\title{
M2-branes, 3-Lie Algebras and Plücker relations
}

\author{
G. Papadopoulos \\ Department of Mathematics \\ King's College London \\ Strand \\ London WC2R 2LS, UK
}

\begin{abstract}
We find that the structure constants 4-form of a metric 3-Lie algebra is the sum of the volume forms of orthogonal 4-planes proving a conjecture in math/0211170. In particular, there is no metric 3-Lie algebra associated to a $\mathfrak{u}(N)$ Lie algebra for $N>2$. We examine the implication of this result on the existence of a multiple M2-brane theory based on metric 3-Lie algebras.
\end{abstract}




\section{Introduction}

The Jacobi identity of a metric 3 -Lie algebra, $\mathfrak{a}_{[3]}$, is

$$
F_{H[A B C} F_{D] E M}^{H}=0, \quad A, B, C, \cdots=0, \ldots, n-1
$$

where $F$ are the structure constants and $n$ is the dimension of $\mathfrak{a}_{[3]}$, respectively. Compatibility with a metric requires that the structure constants $F$ are skew-symmetric in all indices $^{1}$. The generalization of (1.1) to metric $k$-Lie algebras is apparent. In particular for $k=2$, (1.1) becomes the Jacobi identity of a standard metric Lie algebra.

Apart from the interpretation of (1.1) as a Jacobi identity for a 3-Lie algebra, it can also be thought of as a generalized Plücker relation. The latter interpretation for the analogous relation for 4-Lie algebras has been instrumental for the classification of maximally supersymmetric backgrounds in IIB supergravity [1]. Moreover, it was conjectured $^{2}$ in [2] that the only solutions to Jacobi identity are those for which $F$ is the sum of the volume forms of orthogonal $(k+1)$-planes. This was verified for all metric 3-Lie algebras and metric 4-Lie algebras up to and including dimensions 8 and 10, respectively.

More recently, a theory was proposed by Bagger and Lambert [3] for multiple M2branes based on metric 3-Lie algebras. Later it was supersymmetrized by Gustavsson in [4], see also [5]. This followed earlier attempts to construct superconformal $\mathcal{N}=8$ Chern-Simons [6] and multiple M2-brane theories [7]. The relation of the multiple M2branes theory to the maximally supersymmetric gauge theory which describes multiple D2-branes has been investigated in $[8,9,10,11]$ and some other aspects have been examined in $[12,13,14,15,16,17]$. Despite the progress that has been made towards understanding the proposed multiple M2-brane theory based on metric 3-Lie algebras, there are no known solutions other than those given in [2], see also [18].

The main result of this paper is to prove the conjecture of [2] for metric 3-Lie algebras, ie the structure constants of a metric 3-Lie algebra, $\mathfrak{a}_{[3]}$ with Euclidean signature ${ }^{3}$, can be written as

$$
F=\sum_{r} \mu^{r} d \operatorname{vol}\left(V_{r}\right), \quad V_{r} \subset \mathfrak{a}_{[3]}
$$

where the 4-planes $V_{r}$ and $V_{r^{\prime}}$ are mutually orthogonal for $r \neq r^{\prime}$ and $\mu^{r}$ are constants. This conjecture for metric 3-Lie algebras has also appeared more recently in $[4,18,15]$.

To prove our result, first observe that given a vector $X$ in $\mathfrak{a}_{[3]}$, one can associate a metric Lie algebra $\mathfrak{a}_{[2]}(X)$ to $\mathfrak{a}_{[3]}$ defined as the orthogonal complement of $X$ in $\mathfrak{a}_{[3]}$ with structure constants $i_{X} F$. It is easy to verify that $i_{X} F$ satisfy the Jacobi identity using (1.1). Then we demonstrate the following two statements:

- If $\mathfrak{a}_{[3]}$ admits an associated Lie algebra $\mathfrak{a}_{[2]}(X)$ and $\mathfrak{a}_{[2]}(X)$ does not have a biinvariant 4 -form, then $F$ is volume form of a 4 -plane.

\footnotetext{
${ }^{1}$ We raise and lower indices using the compatible metric. Since there is a metric, we do not distinguish between a vector space and its dual.

${ }^{2}$ In the published version of [2] a weaker statement for this conjecture has appeared. The conjecture in this form can be found in the first version of [2], page 3, see arXiv.

${ }^{3}$ In what follows, we assume that the metric on $\mathfrak{a}_{[3]}$ is Euclidean unless it is explicitly stated otherwise.
} 
- If all the associated metric Lie algebras of $\mathfrak{a}_{[3]}$ are $\mathfrak{a}_{[2]}(X)=\oplus^{\ell} \mathfrak{u}(1) \oplus \mathfrak{s s}$, for some $\ell \geq 0$, where $\mathfrak{s s}$ is a semi-simple Lie algebra which commutes with $\oplus^{\ell} \mathfrak{u}(1)$, then $F$ is as in (1.2).

We complete the proof by showing that all metric Lie algebras are isomorphic to $\oplus^{\ell} \mathfrak{u}(1) \oplus$ $\mathfrak{s s}$ in appendix A.

This paper is organized as follows. In section two, we give the proof of the first statement. In section 3, we give the proof of the second statement and in section 4, we examine the applications of our results in the context of multiple M2-branes. In appendix A, we demonstrate that all metric Lie algebras are isomorphic to $\oplus^{\ell} \mathfrak{u}(1) \oplus \mathfrak{s s}$.

\section{3-Lie algebras and invariant 4 -forms}

The Jacobi identity of metric $k$-Lie algebras is an over-constrained quadratic equation for the structure constants $F$. In particular, it is easy to see that the ratio of the number of relations given by the Jacobi identity to the number of components of the structure constants $F$ grows as $n^{k-1}$ for large $n$. In particular, it grows linearly for Lie algebras and quadratically for 3-Lie algebras. So it is clear that the structure constants of $k$-Lie algebras for $k>2$ are more restricted than those of standard Lie algebras.

To prove the first statement in the introduction, without loss of generality, take the vector field $X$ to be along the 0 direction. Then split the indices as $A=(0, i), B=(0, j)$ and so on, with $i, j, \cdots=1, \ldots, n-1$. Setting $A=M=0$ and the rest of the free indices in the range $1, \ldots, n-1$ in (1.1), it is easy to see that

$$
f_{i j k}=F_{0 i j k}
$$

satisfy the Jacobi identity of standard Lie algebras and $f$ are the structure constants of $\mathfrak{a}_{[2]}(X)$. Thus we have written $F$ as

$$
F=\frac{1}{3 !} f_{i j k} e^{0} \wedge e^{i} \wedge e^{j} \wedge e^{k}+\frac{1}{4 !} \phi_{i j k l} e^{i} \wedge e^{j} \wedge e^{k} \wedge e^{l}
$$

where $\left(e^{0}, e^{i}\right), i=1, \ldots, n-1$, is an orthonormal basis.

Next set $M=0$ and the rest of the free indices in the range $1, \ldots, n-1$ in (1.1). Using the skew-symmetry of $F$, one finds that

$$
\phi_{h[i j k} f_{d] e}^{h}=0 .
$$

This implies that the 4 -form $\phi$ is bi-invariant with respect to $\mathfrak{a}_{[2]}(X)$. Since by assumption such form cannot exist, we conclude that $\phi=0$. Thus, we find so far that

$$
F=\frac{1}{3 !} f_{i j k} e^{0} \wedge e^{i} \wedge e^{j} \wedge e^{k} .
$$

Next taking all free indices in (1.1) in the range $1, \ldots, n-1$, we find that

$$
f_{[i j k} f_{h] d e}=0 .
$$


This is the classical Plücker relation which implies that $f$ is a simple ${ }^{4}$ form. Thus one concludes that the only solution to (1.1) is

$$
F=\mu e^{0} \wedge e^{1} \wedge e^{2} \wedge e^{3},
$$

for some constant $\mu$, where we have chosen the four 1-forms, without loss of generality, to lie in the first four directions. This proves the first statement of the paper.

The assumption that $\mathfrak{a}_{[2]}(X)$ does not admit a bi-invariant 4 -form is not very strong. To see this, it is known that bi-invariant forms on the Lie algebra of a group give rise to parallel forms with respect to the Levi-Civita connection on the associated simply connected group manifold. So if $\mathfrak{a}_{[2]}(X)$ admits a bi-invariant 4 -form, the associated group manifold $G$ admits a parallel 4 -form which is necessarily harmonic. So for compact Lie groups, parallel 4-forms represent non-trivial classes in the 4-th deRham cohomology of $G$. Thus if a compact Lie group admits a parallel 4 -form, then $H_{d R}^{4}(G) \neq 0$. However for a large class of Lie groups, which includes all semi-simple ones, $H_{d R}^{4}(G)=0$. Thus we conclude that if an associated Lie algebra $\mathfrak{a}_{[2]}(X)$ to $\mathfrak{a}_{[3]}$ is semi-simple, then the assumption of the first statement in the introduction is satisfied and the only solution is as in (2.6).

\section{3-Lie algebras and Lie algebras}

To proceed to prove the second statement, first observe that we have already demonstrated the result for $\ell=0$ at the end of the previous section. Next, let us consider the case that for some $X$ the associated Lie algebra is

$$
\mathfrak{a}_{[2]}(X)=\mathfrak{u}(1) \oplus \mathfrak{s} \mathfrak{s}
$$

In such as case, $H_{d R}^{4}(G) \neq 0$ and there are bi-invariant 4-forms on $\mathfrak{a}_{[2]}(X)$. Such biinvariant 4-forms are of the type

$$
\phi=e^{n-1} \wedge \varphi=\frac{1}{3 !} \varphi_{\alpha \beta \gamma} e^{n-1} \wedge e^{\alpha} \wedge e^{\beta} \wedge e^{\gamma}, \quad \alpha, \beta, \gamma=1, \ldots, n-2,
$$

where $\varphi$ is a bi-invariant form on the semi-simple part $\mathfrak{s s}$ of $\mathfrak{a}_{[2]}(X)$. Without loss of generality, we have chosen the $\mathfrak{u}(1)$ direction along $e^{n-1}$. Thus so far we have

$$
F=\frac{1}{3 !} f_{\alpha \beta \gamma} e^{0} \wedge e^{\alpha} \wedge e^{\beta} \wedge e^{\gamma}+\frac{1}{3 !} \phi_{\alpha \beta \gamma} e^{n-1} \wedge e^{\alpha} \wedge e^{\beta} \wedge e^{\gamma} .
$$

Since $\varphi$ is a bi-invariant 3 -form on a semi-simple Lie algebra, $\varphi$ is a linear combination of the structure constants $f_{r}$ of the simple Lie algebras ${ }^{5}$ in $\mathfrak{s s}=\oplus^{r} \mathfrak{s}_{r}$. Thus $F$ can be rewritten as

$$
F=\sum_{r}\left(\mu^{r} e^{0}+\nu^{r} e^{n-1}\right) \wedge f_{r}
$$

\footnotetext{
${ }^{4}$ The term "simple" is used in two different ways. In the context of Lie algebras it means the usual simple Lie algebras like $\mathfrak{s u}$ and $\mathfrak{s o}$. In the context of forms it is used in the Plücker sense, ie a $p$-form is simple iff it is the wedge product of $p$ 1-forms, see eg [19].

${ }^{5}$ Every semi-simple Lie algebra decomposes into an orthogonal sum of simple Lie algebras, $\mathfrak{s i}=\oplus^{r} \mathfrak{s}_{r}$, with respect to the bi-invariant inner product, such that $\left[\mathfrak{s}_{r}, \mathfrak{s}_{r^{\prime}}\right]=0$ for $r \neq r^{\prime}$.
} 
where $\mu^{r} \neq 0$ and $\nu^{r}$ are some constants.

Next using that $f_{r}$ and $f_{r^{\prime}}$, for $r \neq r^{\prime}$, are mutually orthogonal, the Jacobi identity (1.1) implies that $\mu^{r} e^{0}+\nu^{r} e^{n-1}$ and $\mu^{r^{\prime}} e^{0}+\nu^{r^{\prime}} e^{n-1}$ are mutually orthogonal as well. This can be easily seen by setting $A, B, C$ to take values in the $\mathfrak{s}_{r}$ and $D, E, M$ to take values in the $\mathfrak{s}_{r^{\prime}}$ component of $\mathfrak{s s}$, respectively. Moreover applying the Jacobi (1.1) for the same simple component, ie allowing all $A, B, C, D, E, M$ to take values in the same simple component $\mathfrak{s}_{r}$, one finds that $f_{r}$ satisfies the standard Plücker relation, as in (2.5), and so $f_{r}$ must be a simple 3 -form. In conclusion, $F$ is the sum of volume forms of at most two orthogonal 4-planes, ie without loss of generality, it can be written as

$$
F=\mu e^{0} \wedge e^{1} \wedge e^{2} \wedge e^{3}+\nu e^{4} \wedge e^{5} \wedge e^{6} \wedge e^{7}
$$

where $\mu$ and $\nu$ are constants.

This can be extended to metric 3-Lie algebras for which all associated Lie algebras are

$$
\mathfrak{a}_{[2]}(X)=\oplus^{\ell} \mathfrak{u}(1) \oplus \mathfrak{s s}, \quad \ell>1
$$

To begin write $F=e^{0} \wedge f+\phi$ as in (2.2), where $f$ are the structure constants of $\mathfrak{s s}$ and $\phi$ satisfies (2.3). Since semi-simple Lie algebras do not admit bi-invariant 1-, 2- and 4-forms, see eg [20], and $\oplus^{\ell} \mathfrak{u}(1)$ commutes with $\mathfrak{s s}$, the most general invariant 4-form $\phi$ which satisfies $(2.3)$ is

$$
\phi=\sum_{I} \rho^{I} \wedge \varphi_{I}+\xi
$$

where $\rho^{I}$ are the 1 -forms along the $\oplus^{\ell} \mathfrak{u}(1)$ directions, $\varphi_{I}$ are bi-invariant 3 -forms of $\mathfrak{s s}$ and $\xi$ is a 4 -form along the $\oplus^{\ell} \mathfrak{u}(1)$ directions.

Since the bi-invariant 3 -forms of semi-simple Lie algebras are linear combinations of those associated with the structure constants of the simple components $\mathfrak{s}_{r}$, we have

$$
\varphi_{I}=\nu_{I}^{r} f_{r}
$$

So $F$ can be rewritten as

$$
F=\sum_{r} \sigma^{r} \wedge f_{r}+\xi
$$

for some constants $\mu^{r} \neq 0$ and $\nu_{I}^{r}$, where

$$
\sigma^{r}=\mu^{r} e^{0}+\sum_{I} \nu_{I}^{r} \rho^{I}
$$

Using that $f_{r}$ and $f_{r^{\prime}}$, for $r \neq r^{\prime}$, are mutually orthogonal, the Jacobi identity (1.1) implies that $\sigma^{r}$ and $\sigma^{r^{\prime}}$ are mutually orthogonal as well. Thus there is an orthogonal transformation in $\oplus^{\ell} \mathfrak{u}(1)$ such that $F$ can be written, without loss of generality, as

$$
F=\sum_{r} \lambda_{r} e^{r} \wedge f_{r}+\xi
$$


for some constants $\lambda_{r}$, where $e^{r}$ belong to an orthonormal basis in the $\oplus^{\ell} \mathfrak{u}(1)$ directions, ie in particular $e^{r} \perp e^{r^{\prime}}$ for $r \neq r^{\prime}$ and $i_{e^{r}} f_{s}=0$ for all $r$ and $s$. As in previous cases, allowing all indices $A, B, C, D, E, M$ of (1.1) to take values in the same simple component $\mathfrak{s}_{r}$, one finds that $f_{r}$ satisfies the standard Plücker relation, (2.5), and so $f_{r}$ must be a simple 3 -form. Thus the component of $F$ orthogonal to $\xi$ is as in (1.2).

Furthermore, using the orthogonality of $f_{r}$ and $\xi$ and the Jacobi identity (1.1), one finds that

$$
i_{e^{r}} \xi=0
$$

This can be easily seen from (1.1) by setting $A, B, C$ to take values in the $\mathfrak{s}_{r}$ and $D, E, M$ to take values in the $\oplus^{\ell} \mathfrak{u}(1)$. A consequence of (3.12) is that the 4-form $\xi$ on $\oplus^{\ell} \mathfrak{u}(1)$ satisfies (1.1), ie $\oplus^{\ell} \mathfrak{u}(1)$ is also a metric 3-Lie algebra, $\mathfrak{b}_{[3]}$, with structure constants $\xi$. Since the dimension of $\mathfrak{b}_{[3]} \subset \mathfrak{a}_{[3]}$ is strictly less than that of the original metric 3-Lie algebra $\mathfrak{a}_{[3]}$, the analysis can be repeated and it will terminate after a finite number of steps. To summarize, we have demonstrated (1.2) under the assumption that all the associated Lie algebras $\mathfrak{a}_{[2]}(X)$ of $\mathfrak{a}_{[3]}$ are isomorphic to $\oplus^{\ell} \mathfrak{u}(1) \oplus \mathfrak{s s}$.

To prove the conjecture in [2] for metric 3-Lie algebras, it remains to show that all metric Lie algebras are isomorphic to $\oplus^{\ell} \mathfrak{u}(1) \oplus \mathfrak{s s}$. This is done in appendix $A$ and so we establish (1.2).

\section{Multiple M2-branes}

Some of the results we have presented for metric 3-Lie algebras can be extended to metric $\mathrm{k}$-Lie algebras for $k>3$. This together with the $n^{k-1}$ growth of relations on the structure constants mentioned in section 2, strengthens the conjecture in [2]. In particular, it looks likely that the structure constants of metric k-Lie algebras are sums of volume forms of orthogonal $(k+1)$-planes. Though there is not a proof for this for $k>3$. Our result also relies on the existence of a Euclidean inner product on the 3-Lie algebras. Thus the Lorentzian signature case has to be examined separately. As it has already been mentioned in [2], it is unlikely that (1.2) holds for other signatures.

A consistency condition for the validity of a multiple M2-brane theory for $N \geq 2$ is the derivation from it of the maximally supersymmetric gauge theory in 3-dimensions which describes $N$ coincident D2-branes. The gauge group of the latter for D2-branes in flat space is $U(N)$. Our result demonstrates that the expected $U(N)$ gauge group cannot be recovered from a metric 3-Lie algebra for $N>2$. This in particular includes the identification of the gauge group $U(N)$ in a metric 3 -Lie algebra as in eg $[8,9,10]$. So the consistency check on the multiple M2-brane theory with that of D2-branes cannot be met for $N>2$. In fact our result excludes all other possibilities for other gauge groups apart from $\times{ }^{\ell} S U(2)$.

The above results clearly indicate that some of the assumptions must be weakened. This has already been anticipated by Gran, Nilsson and Petersson in [11] where they considered a theory of multiple M2-branes using an $F$ which is not skew-symmetric. From this theory one can consistently derive the effective theory of $N$ coincident D2branes. However, this multiple M2-brane theory is not described by an action. The 
dynamics of the theory is given in terms of field equations. Though one may be able to define a Hamiltonian and so quantize the theory. Alternatively, one can consider multiple M2-brane theories based on Lorentzian metric 3-Lie algebras. Again, it is not apparent that such a choice will lead to a consistent theory. First, the possibility remains open that $F$ may again be written as (1.2). Some partial results in [2] indicate this. In addition, the Hamiltonian of the theory will be unbounded from below which in turn may lead to difficulties with the particle physics interpretation of the theory.

\section{Acknowledgements}

I would like to thank U Gran for many helpful discussions and comments.

\section{Appendix A Metric Lie algebras}

It is known that any Lie algebra $\mathfrak{g}$, which is not semi-simple, contains an invariant (normal) solvable subalgebra, $\mathfrak{r}$, called the radical, such that $\mathfrak{g} / \mathfrak{r}$ is semi-simple, see eg [21] page 234. This follows from the definition of semi-simple Lie algebras. Now assume that $\mathfrak{g}$ admits a Euclidean invariant metric $B$. In such case, one can define the semisimple algebra $\mathfrak{s s}$ as the orthogonal complement of $\mathfrak{r}$ in $\mathfrak{g}$. Using this and the invariance property of $B$ which schematically can be written as

$$
B([\mathfrak{g}, \mathfrak{g}], \mathfrak{g})+B(\mathfrak{g},[\mathfrak{g}, \mathfrak{g}])=0
$$

one finds that

$$
[\mathfrak{s s}, \mathfrak{s s}] \subseteq \mathfrak{s} \mathfrak{s}, \quad[\mathfrak{s s}, \mathfrak{r}] \subseteq \mathfrak{r}, \quad[\mathfrak{r}, \mathfrak{r}] \subseteq \mathfrak{r}
$$

So to prove the statement, we have first to show that for metric Lie algebras $\mathfrak{g}, \mathfrak{r}$ is abelian. From the definition of solvable algebras there is an $n$ such that $\mathfrak{r}^{n}$ is an abelian Lie algebra, where $\mathfrak{r}^{i+1}=\left[\mathfrak{r}^{i}, \mathfrak{r}^{i}\right]$ and $\mathfrak{r}^{0}=\mathfrak{r}$. The metric $B$ restricted on $\mathfrak{r}$ and $\mathfrak{r}^{n}$ is not degenerate. Since $\mathfrak{r}^{n}$ abelian, it is easy to see that

$$
B\left(\left[\mathfrak{r}^{n}, \mathfrak{r}^{n}\right], \mathfrak{r}^{n-1}\right)+B\left(\mathfrak{r}^{n},\left[\mathfrak{r}^{n}, \mathfrak{r}^{n-1}\right]\right)=B\left(\mathfrak{r}^{n},\left[\mathfrak{r}^{n}, \mathfrak{r}^{n-1}\right]\right)=0
$$

So if $\left[\mathfrak{r}^{n-1}, \mathfrak{r}^{n}\right] \neq\{0\}$, it is orthogonal to the whole of $\mathfrak{r}^{n}$. But $\left[\mathfrak{r}^{n-1}, \mathfrak{r}^{n}\right] \subseteq \mathfrak{r}^{n}$ and since the metric is not degenerate, one concludes that $\left[\mathfrak{r}^{n-1}, \mathfrak{r}^{n}\right]=0$. Similarly

$$
B\left(\left[\mathfrak{r}^{n-1}, \mathfrak{r}^{n}\right], \mathfrak{r}^{n-1}\right)+B\left(\mathfrak{r}^{n},\left[\mathfrak{r}^{n-1}, \mathfrak{r}^{n-1}\right]\right)=B\left(\mathfrak{r}^{n},\left[\mathfrak{r}^{n-1}, \mathfrak{r}^{n-1}\right]\right)=0
$$

But $\left[\mathfrak{r}^{n-1}, \mathfrak{r}^{n-1}\right]=\mathfrak{r}^{n} \neq\{0\}$. Since the metric is not degenerate, one concludes that $\left[\mathfrak{r}^{n-1}, \mathfrak{r}^{n-1}\right]=0$. Therefore $\mathfrak{r}^{n-1}$ is also abelian and so $\mathfrak{r}^{n-1}=\mathfrak{r}^{n}$. Continuing in this way, one can show that $\mathfrak{r}=\mathfrak{r}^{n}$ is abelian. So without loss of generality, we can write $\mathfrak{r}=\oplus^{\ell} \mathfrak{u}(1)$.

It remains to show that $[\mathfrak{s s}, \mathfrak{r}]=0$. This follows again from the invariance of the metric. Indeed

$$
B([\mathfrak{r}, \mathfrak{s s}], \mathfrak{r})+B(\mathfrak{s s},[\mathfrak{r}, \mathfrak{r}])=B([\mathfrak{r}, \mathfrak{s s}], \mathfrak{r})=0
$$


using that $\mathfrak{r}$ is abelian. Thus if $[\mathfrak{r}, \mathfrak{s s}] \neq\{0\}$, the subspace $[\mathfrak{r}, \mathfrak{s s}]$ of $\mathfrak{r}$ is orthogonal to the whole $\mathfrak{r}$. Since the metric is non-degenerate, one again concludes that $[\mathfrak{r}, \mathfrak{s s}]=0$. Thus all metric Lie algebras can be written as $\oplus^{\ell} \mathfrak{u}(1) \oplus \mathfrak{s s}$ with $\mathfrak{s s}$ to commute with $\oplus^{\ell} \mathfrak{u}(1)$.

\section{References}

[1] J. Figueroa-O'Farrill and G. Papadopoulos, "Maximally supersymmetric solutions of ten- and eleven-dimensional supergravities," JHEP 0303 (2003) 048 [arXiv:hepth/0211089].

[2] J. Figueroa-O'Farrill and G. Papadopoulos, "Pluecker-type relations for orthogonal planes," arXiv:math/0211170.

[3] J. Bagger and N. Lambert, "Modeling multiple M2's," Phys. Rev. D 75, 045020 (2007) [arXiv:hep-th/0611108].

[4] A. Gustavsson, "Algebraic structures on parallel M2-branes," arXiv:0709.1260 [hepth].

"Selfdual strings and loop space Nahm equations," arXiv:0802.3456 [hep-th].

[5] J. Bagger and N. Lambert, "Gauge Symmetry and Supersymmetry of Multiple M2Branes," Phys. Rev. D 77, 065008 (2008) [arXiv:0711.0955 [hep-th]].

[6] J. H. Schwarz, "Superconformal Chern-Simons theories," JHEP 0411 (2004) 078 [arXiv:hep-th/0411077].

[7] A. Basu and J. A. Harvey, "The M2-M5 brane system and a generalized Nahm's equation," Nucl. Phys. B 713 (2005) 136 [arXiv:hep-th/0412310].

[8] S. Mukhi and C. Papageorgakis, "M2 to D2," arXiv:0803.3218 [hep-th].

[9] N. Lambert and D. Tong, "Membranes on an Orbifold," arXiv:0804.1114 [hep-th].

[10] J. Distler, S. Mukhi, C. Papageorgakis and M. Van Raamsdonk, "M2-branes on M-folds," arXiv:0804.1256 [hep-th].

[11] U. Gran, B. E. W. Nilsson and C. Petersson, "On relating multiple M2 and D2branes," arXiv:0804.1784 [hep-th].

[12] D. S. Berman, L. C. Tadrowski and D. C. Thompson, "Aspects of Multiple Membranes," arXiv:0803.3611 [hep-th].

[13] M. Van Raamsdonk, "Comments on the Bagger-Lambert theory and multiple M2branes," arXiv:0803.3803 [hep-th].

[14] A. Morozov, "On the Problem of Multiple M2 Branes," arXiv:0804.0913 [hep-th].

[15] P. M. Ho, R. C. Hou and Y. Matsuo, "Lie 3-Algebra and Multiple M2-branes," arXiv:0804.2110 [hep-th]. 
[16] J. Gomis, A. J. Salim and F. Passerini, "Matrix Theory of Type IIB Plane Wave from Membranes," arXiv:0804.2186 [hep-th].

[17] E. A. Bergshoeff, M. de Roo and O. Hohm, "Multiple M2-branes and the Embedding Tensor," arXiv:0804.2201 [hep-th].

[18] M. A. Bandres, A. E. Lipstein and J. H. Schwarz, "N = 8 Superconformal ChernSimons Theories," arXiv:0803.3242 [hep-th].

[19] P. Griffiths and J. Harris, "Principles of Algebraic Geometry," John Wiley 1978.

[20] M. Mimura and H. Toda, "Topology of Lie Groups, I and II," American Mathematical Society Vol 91, (1991).

[21] R. Gilmore, "Lie groups, Lie algebras, and some of their applications," Krieger, Florida (1974). 